\title{
Indicadores de desempenho de bibliotecas no campo da saúde: relato de estudo piloto na Fiocruz
}

Maria Cristina S. Guimarães;

Doutora em Ciência da Informação;

CICT/FIOCRUZ - cguima@cict.fiocruz.br

Etiene Gonzalez Lins;

Bibliotecária; Bolsista PROGESTÃo;

FIOCRUZ/FAPERJ - etiene.lins@gmail.com

Jeorgina Gentil Rodrigues;

Bibliotecária; Mestre em Ciência da Informção;

CICT/FIOCRUZ - jeorgina@cict.fiocruz.br

Camila Clementino Lamarão;

Socióloga; Bolsista PROGESTÃo;

FIOCRUZ/FAPERJ - gricss@gmail.com

Márcia Jabor Santos;

Mestre em Ciência da Informação; Professora aposentada da UFF - japorgarcia@gmail.com

Vânia Guerra da Silva;

Bibliotecária; Especialista em Informação

Científica e Tecnológica em Saúde;

CICT/FIOCRUZ - vguerra@cict.fiocruz.br

Diones Ramos da Silva.

Bibliotecária; CICT/FIOCRUZ -

diones@cict.fiocruz.br

Relato de projeto de pesquisa, em curso, sobre a implantação de um programa de avaliação de desempenho de bibliotecas no campo da saúde. Uma equipe de pesquisadores e bibliotecários tomou como ponto de partida a Norma ISO 11620 para derivar um conjunto mínimo de indicadores adequados à avaliação de três diferentes bibliotecas especializadas da Fundação Oswaldo Cruz. O relato enfatiza a importância do 
processo de cooperação entre os profissionais, ingrediente essencial para sustentabilidade da iniciativa e os desafios futuros que se avizinham.

Palavras-Chave: Indicadores de desempenho; Avaliação de serviços de bibliotecas; ISO 11620.

\section{Performance Indicators: a pilot study in FIOCRUZ health and biomedical libraries}

This paper describes the recent efforts of a science and technology health institute to implement a library services evaluation program. Based on the ISO 11620 a team of researchers and librarians have considered a set of performance indicators to be used in its three specialized libraries. The way taken so far and the collaborative process that have been shaping the whole process are described; future challenges are also indicated.

Key-words: Library services evaluation; Performance indicators; ISO 11620.

Recebido em 15.02.2006 Aceito em 28.02.2007

\section{Introdução}

Os serviços de informação, e mais especialmente as bibliotecas, chegaram ao século XXI com um amplo leque de oportunidades, mas também de desafios. A sociedade contemporânea tem como marca principal um casamento virtuoso entre tecnologias e conteúdos, sendo que o imperativo da velocidade e do instantâneo provoca uma aparente dissociação lógica entre a informação e o documento. Na mesma medida em que especialistas discutem o futuro dos livros como os conhecemos hoje, às bibliotecas cabe o imperativo de se re-pensar enquanto lócus privilegiado de estoque e acesso à informação codificada, independente do documento. A dinâmica atual do empreendimento científico, o volume crescente de novos títulos de periódicos, representativos de novas especialidades e temáticas, o uso intensivo de tecnologias de informação e comunicação -TICs - , usuários cada vez mais exigentes com a qualidade de serviços oferecidos e o tempo de resposta, e gestores e tomadores de decisão mais ciosos e preocupados com decisões mais acertadas e com os 
custos de manutenção de acervos dispendiosos, em tempos de recursos financeiros escassos, compõem o quadro de alguns dos maiores desafios colocados à gestão das bibliotecas.

Esses desafios colocam para os serviços de informação, como para todas as organizações, o imperativo do planejamento, atividade vital na busca de maximizar os recursos disponíveis e obter o melhor proveito das tecnologias disponíveis. O investimento contínuo em inovação deve ter seu foco não só nas práticas e estruturas técnicas mas, também, naquelas organizacionais e humanas, dentro de um ambiente onde as orientações estratégicas devem ser claras e objetivas: o principal ativo de uma biblioteca é o usuário, e não o acervo.

Um dos mais valiosos instrumentos-guia para as atividades de planejamento nas bibliotecas são as avaliações de desempenho. Por desempenho entende-se o grau de atendimento dos objetivos propostos, particularmente em termos das necessidades dos usuários. A medida de desempenho deve indicar se a biblioteca está atendendo ao planejado, a partir de uma coleção de dados estatísticos.

Desde meados do século passado, e especialmente na GrãBretanha e nos Estados Unidos da América, o interesse na área de avaliação levou ao desenvolvimento e divulgação de uma grande variedade de estudos e abordagens sobre medidas de desempenho. Nasceram daí orientações metodológicas diversas, com focos e recortes em diferentes atividades das bibliotecas, produzindo um amplo conjunto de indicadores de desempenho. Essas diferentes abordagens em muito contribuíram para o entendimento dos diferentes contextos e processos de gestão de bibliotecas, mas pouco tem contribuído para produzir estudos comparativos. A situação se torna mais desafiante para o caso de diferentes bibliotecas especializadas ligadas a uma mesma instituição.

De fato, não só as medidas de desempenho guardam singularidades inerentes a cada biblioteca individualmente, como também as mesmas devem ser consideradas como um instrumento para o processo de avaliação que, inegavelmente, envolve julgamentos de valor. Dispor de instrumentos de padronização para coleta de dados e desenvolvimento de indicadores é então uma atividade fundamental para assegurar, na justa medida técnica, que o processo de avaliação seja o mais transparente e eqüitativo possível entre diferentes bibliotecas.

Em resposta a essa necessidade de padronização, a ISO International Standard Organization, ainda no começo dos anos noventa, se debruçou sobre o desafio da criação de padrões para medida de desempenho das bibliotecas, o que resultou na Norma ISO 11620/98 (Information and Documentation - Library Performance Indicators). Esse esforço nasceu em um contexto mundial de globalização, de integração de blocos regionais, de disputa internacional por fundos de pesquisa, e não menos importante, de cooperação internacional entre bibliotecas.

Esse é também o cenário onde se situa o Cict - Centro de Informação Científica e Tecnológica, Unidade Técnica da Fundação Oswaldo Cruz - Fiocruz, instituição de reconhecida competência 
internacional no campo da pesquisa, desenvolvimento, produção e formação de recursos humanos em saúde e ciências biomédicas. Atualmente, para atender pesquisadores nessas diferentes áreas, são dez as bibliotecas que ofertam informação e serviços para tão variadas atividades. Cada biblioteca se insere em um contexto impar, possui um acervo diferenciado e desenvolve atividades particulares com vistas a atender um grupo de usuários com perfil específico e necessidades próprias.

A prática da avaliação, através de medidas de desempenho, ainda que reconhecida e abraçada como atividade primordial para planejamento e gestão coordenada das bibliotecas, ainda carece de uma visão orgânica e integradora. Paralelamente, em tempos de recursos financeiros escassos, eficiência e eficácia são conceitos chave para produzir evidências para o tomador de decisão. A padronização das medidas de desempenho, tomando a ISO 11620 como ponto de partida, foi a estratégia adotada na busca de harmonia entre as métricas atuais de cada biblioteca. Ainda que se reconheça as limitações da referida Norma, é entendimento que representa um primeiro e fundamental passo a ser dado pela instituição.

O presente trabalho relata o desenho e os primeiros passos de um projeto de pesquisa em andamento, ressaltando o ambiente de cooperação e colaboração que vêm propiciando uma discussão frutífera e um estratégico processo de aprendizagem.

\section{Medidas de desempenho e avaliação de serviços}

O dinamismo e a velocidade das transformações sociais que caracterizam a sociedade contemporânea requerem das instituições celeridade nas respostas e flexibilidade para adaptar-se a novas e desafiantes situações. Entretanto, há sempre que se buscar sustentabilidade nas mudanças e inovações. Lancaster (1996, p. 3) ressalta que "(...) crescimento saudável implica adaptação a condições constantemente mutáveis, e adaptação implica avaliação para determinar que mudanças precisam ser feitas e qual a melhor maneira de realizá-las".

A avaliação é, ou deve ser o processo que gera evidências e fornece ao tomador de decisão informações que permitem justificar as ações presentes e orientações futuras. Nesse sentido, a avaliação é uma etapa necessária tanto para os gestores quanto para os profissionais de informação/bibliotecários: para os primeiros, deve ser capaz de responder pela eficácia e eficiência dos investimentos feitos, para os segundos, deve explicitar a qualidade e bom desempenho de suas atuações.

Mandani (2004) alerta para a importância da clareza conceitual quando se trata com o processo de avaliação. O primeiro esclarecimento deve ser feito entre medida de desempenho e avaliação. A primeira é uma medida de uma atividade ou de um serviço. Mais, a medida de desempenho é um instrumento por sobre o qual julgamentos são feitos no curso de um processo de avaliação. O desempenho, por si só, não é bom 
ou ruim, positivo ou negativo, ele é o que é. Somente frente a objetivos propostos, a metas a serem alcançadas, é que um desempenho pode ser aferido. Assim, qualquer avaliação depende de um objetivo e de uma medida de desempenho. O indicador, finalmente, é uma expressão (numérica, simbólica ou semântica) que caracteriza atividades (objetos, pessoas, eventos) tanto quantitativa ou qualitativamente, com vistas a aferir o valor dessas atividades. Qualidade das fontes de informação, relação terminais de consulta/número de usuários, satisfação dos usuários são exemplos de indicadores usados em avaliações de serviço de informação.

Em artigo recente, Rozados (2005) faz uma discussão e síntese do uso de indicadores na gestão de recursos de informação. Indicadores de input (entrada), output (saída) e outcomes (resultados) formam a tipologia mais conhecida de indicadores, e expressam a visão sistêmica de uma biblioteca. Dos três, os indicadores de resultados representam os melhores esforços para aferir a qualidade e o impacto dos serviços prestados, e coloca o usuário no centro de atenção das metodologias de avaliação.

A avaliação de desempenho, por seu lado, engloba a coleção e análise de dados estatísticos para retratar o desempenho de uma biblioteca, ou seja, uma medida de comparação entre o que está sendo feito (desempenho), com o que deveria ser feito (missão) e com aquilo que se gostaria de fazer (metas). O foco na avaliação de desempenho é, principalmente, resultado da integração de blocos regionais no contexto internacional, especialmente na Europa, de onde nasceu do desafio de implementar metodologia única para avaliar as bibliotecas nacionais, tão peculiares que são na guarda e conservação do patrimônio nacional. Entrou em campo a ISO com a Norma ISO 11620, publicada em 1998, que congrega um conjunto de definições, metodologias e a definição de 29 (vinte e nove) indicadores de desempenho associados a um ou mais métodos de obtenção de dados e análise de resultados. Em 2003 foi publicada uma atualização da referida norma (ISO11620: 1998/Adm.1/2003), com a incorporação de novos indicadores de desempenho.

Enquanto instrumento de padronização, a ISO11620 estabelece um grupo de indicadores para aplicação em todos os tipos de bibliotecas. Além disso, estabelece uma relação entre qualidade de serviços de biblioteca e o tópico mais amplo de administração e garantia de qualidade, uma vez que valida e apóia-se na Norma ISO 9004-2 (INTERNATIONAL ORGANIZATION FOR STANDARDIZATION, 1991). Para cada indicador constam: objetivo, escopo, definição, metodologia, interpretação e fontes de referência e, entre os procedimentos são adotados cálculo de percentual, amostragem, questionário e entrevista. A norma oferece também detalhada orientação sobre a metodologia e análises sobre seu emprego. Alguns serviços e atividades importantes, como, por exemplo, treinamento de usuário e serviços eletrônicos, não estão contemplados, 
mas são cobertos em outra norma, a ISO 2789/2003 (INTERNATIONAL ORGANIZATION FOR STANDARDIZATION, 2003b).

Muitas iniciativas de desenvolvimento de metodologias de avaliação foram empreendidas nos países desenvolvidos nos últimos trinta anos, muitas delas já englobando os serviços eletrônicos. Algumas das mais importantes são citadas: O projeto LibEcon nasceu na Comunidade Européia e congrega atualmente 37 países também da América, Ásia e Austrália. Os dados são coletados de acordo com a ISO2789 e ISO11620, possibilitando a prática de benchmarking. O projeto EQUINOX - Library Performance Measurement and Quality Management System nasceu com o foco em serviços eletrônicos. São 14 indicadores de desempenho acordado entre bibliotecários de seis países europeus, indicadores esses que se somam à Norma ISO11620. LibQUAL $+{ }^{\mathrm{TM}}$ (Libqual+ ${ }^{\mathrm{TM}}$, 2005), iniciativa norte-americana resultado de uma associação entre a Association of Research Libraries - ARL - e a Texas A\&M University - TAMU -, é um instrumento de aferição da qualidade dos serviços em função das percepções e expectativas dos usuários. LibQUAL+ ${ }^{\mathrm{TM}}$ foi desenvolvido a partir do SERVQUAL, reconhecido como primeiro padrão desenvolvido para avaliação da qualidade de serviço no setor comercial, trabalho pioneiro de Berry, Parasuraman e Zeithaml (PARASURAMAN et al, 1985), posteriormente adaptado para aplicação em bibliotecas acadêmicas. $O$ instrumento contempla 22 perguntas que cobrem três dimensões das bibliotecas: o espaço, a prestação de serviços e o controle da informação. O COUNTER - Counting Online Usage of Networked Eletronic -, uma iniciativa internacional que une bibliotecários, editores e distribuidores de publicações e se apóia em iniciativas e padrões que buscam normalizar as estatísticas de acesso à informação via web.

No Brasil, a mais conhecida experiência de grande porte aconteceu na Universidade de São Paulo. O Sistema Integrado de Bibliotecas da Universidade de São Paulo - SIBI/USP, desenvolveu o PAQ - Programa de Qualidade de Produtos e Serviços de Informação - para ser implementado em sua rede de bibliotecas. Englobou duas metodologias diferentes de avaliação, o SERVQUAL e o LibQUAL + ${ }^{\mathrm{TM}}$, ajustadas às características das bibliotecas. No relatório, entre outras importantes conclusões, destaca-se a importância do conhecimento das necessidades do usuário: "No ambiente acadêmico, considera-se que o desconhecimento em relação a essas necessidades pode comprometer as atividades de ensino, pesquisa e a geração de novos conhecimentos" (SAMPAIO, 2001, p. 143)

É reconhecido que a Norma ISO 11620 proporciona as condições requeridas para um salto de qualidade no que tange aos processos de avaliação de bibliotecas. A propósito, eis o que ponderou Carbone (1998, p. 41) analisando-a:

Os bibliotecários não podem mais se limitar a relatar seus serviços somente a partir de dados estatísticos sobre os recursos e atividades da biblioteca. Eles devem provar que atendem às necessidades dos usuários e provar de que maneira e a que ponto os serviços da biblioteca as atendem. 
A ISO11620 sugere ainda que os resultados da aplicação dos indicadores podem ser comparados em tempos diferentes numa mesma biblioteca e podem ser feitas comparações entre bibliotecas, ainda que cautela seja sempre necessária. Para o estabelecimento de novos indicadores (conteúdo informativo, comparabilidade, propriedade e outros) devem ser observados critérios, bem como a sua adequada descrição.

Deve ser enfatizado que o valor dessa Norma reside, sobretudo, no fato de ser aplicável a qualquer tipo de biblioteca e, por isso, prover uma base e linguagem única para o caso de diferentes perfis de bibliotecas acolhidas sob uma mesma coordenação, como é o caso de três diferentes bibliotecas especializadas ligadas ao Centro de Informação Científica e Tecnológica - Cict, unidade técnica da Fundação Oswaldo Cruz - Fiocruz.

\section{0 contexto de estudo - o Cict/Fiocruz}

A Fundação Oswaldo Cruz é uma instituição vinculada ao Ministério da Saúde, e abriga atividades que incluem o desenvolvimento de pesquisas; a prestação de serviços hospitalares e ambulatoriais de referência em saúde; a fabricação de vacinas, medicamentos, reagentes e kits de diagnóstico; o ensino e a formação de recursos humanos; a informação e a comunicação em saúde, ciência e tecnologia; o controle da qualidade de produtos e serviços; e a implementação de programas sociais. Tem como missão promover a saúde e o desenvolvimento social, gerar e difundir conhecimento científico e tecnológico, ser um agente da cidadania. Através de programas de informação e comunicação, vem procurando cumprir papel da maior relevância, o que deu margem, certamente, para que fosse premiado pela Unesco, em 2002, como a instituição do ano na área das ciências.

A Fiocruz guarda em sua história uma relação visceral com a informação. É viva em sua memória, cultura e imaginário a fala de seu patrono e fundador, Oswaldo Cruz, personalidade reconhecida internacionalmente: "Corte-se até a verba para a alimentação. Mas não se sacrifique a biblioteca". Atualmente, a instituição conta com dez bibliotecas distribuídas em diferentes regiões do país, cada uma atendendo a um perfil diferenciado de usuários e suas respectivas necessidades de informação. O comprometimento com a valorização da informação, como ingrediente vital para a pesquisa, é um dos fatores que certamente conduziu, nos anos oitenta, a criação de uma unidade técnica dedicada à guarda, planejamento e gestão da informação científica e tecnológica no campo da saúde.

O Centro de Informação Científica e Tecnológica é responsável por formular políticas, desenvolver estratégias e executar ações de informação e comunicação no campo da ciência e tecnologia em saúde que respondam as demandas da sociedade e do Sistema Único de Saúde SUS -, e de outros órgãos governamentais. Ao Cict estão diretamente 
subordinadas três bibliotecas especializadas, as quais se colocam como foco do presente estudo.

A Biblioteca de Manguinhos - BIBMANG - atua como Centro de Referência em Biomedicina para as instituições de pesquisa no país e oferece suporte ao desenvolvimento do ensino e da pesquisa no âmbito da Fiocruz. Atende ao corpo de docentes, de pesquisadores e alunos de pósgraduação da instituição e da comunidade em geral. A Biblioteca do Instituto Fernandes Figueiras - BIBIFF - está voltada para saúde da mulher, da criança e do adolescente. Suas áreas de especialização são o aleitamento materno, a genética, a ginecologia e a obstetrícia, a infecção hospitalar, a nutrição, a patologia e a pediatria. Seu público-alvo é composto por profissionais de saúde, alunos de pós-graduação, professores, pesquisadores do Instituto Fernandes Figueiras e da rede pública e privada de serviços de saúde. Por fim, a Biblioteca da Escola Nacional de Saúde Pública Sergio Arouca - BIBENSP - atende ao corpo de docentes, pesquisadores e alunos de pós-graduação da Escola Nacional de Saúde Pública - ENSP -, e é aberta também à comunidade em geral. Atua como Centro de Referência a todos os Cursos descentralizados de Saúde Pública desenvolvidos no país e à Rede Pública de Serviços de Saúde no Estado do Rio de Janeiro.

Essas bibliotecas foram criadas em momentos próprios, para atender clientelas e necessidades particulares, e se inserem em contextos singulares de práticas do campo da saúde. A estratégia de planejamento e gestão se faz em um delicado balanço entre autonomia e coordenação central, no reconhecimento das especificidades e na responsabilidade de um todo harmônico que deve ser traduzida, de forma transparente, ao tomador de decisão, especialmente no que diz respeito aos investimentos financeiros. As atividades relacionadas à mensuração de indicadores de desempenho, ainda que realizada anualmente, carece de caráter sistêmico. Da mesma forma, a realização de estudos de satisfação de usuários ainda não está incorporada como instrumento de gestão.

Ciente de suas responsabilidades, e em um ambiente cooperativo, o Cict colocou o desafio de propor uma metodologia padrão para avaliação de desempenho das bibliotecas. A Norma ISO 11620 se colocou então como instrumento estratégico para, em uma primeira etapa, prover uma padronização das métricas de desempenho das bibliotecas.

\section{Projeto - Instrumento de medida de desempenho para as bibliotecas do CICT}

O projeto Instrumento de medida de desempenho para as Bibliotecas do Cict foi iniciado em 2005 com o objetivo principal de propor medidas padrão para o desempenho das três bibliotecas que são coordenadas pelo Cict. A equipe diretamente ligada ao seu desenvolvimento é formada por um coordenador, três bibliotecários (um representante de cada biblioteca) dois bolsistas e um consultor. 
A etapa inicial envolveu uma busca retrospectiva visando à identificação de relatos de experiências prévias com a utilização da Norma ISO 11620, particularmente no Brasil. No contexto brasileiro, nenhuma referência foi localizada. Os documentos recuperados eram enviados em formato eletrônico para todos os membros da equipe, que se reuniam quinzenalmente para discussões técnicas.

Paralelamente, foi realizado um diagnóstico situacional das três bibliotecas que procurou explicitar, para cada uma delas: o perfil dos usuários; os serviços e produtos oferecidos; a infra-estrutura e os recursos humanos disponíveis visando a identificação fatores relevantes para avaliação. Esse diagnóstico foi de fundamental importância para ressaltar as semelhanças e dessemelhanças entre as três bibliotecas, favorecendo um re-conhecimento dos contextos e das práticas individuais.

Esse diagnóstico foi também um pré-requisito para a realização da primeira oficina de trabalho que propiciou a efetiva implantação do projeto, na medida em que esse encontro, incluindo a direção da instituição, foi palco de alianças e compromissos primeiros entre os profissionais. A oficina teve como objetivo a discussão coletiva do conjunto de indicadores propostos pela Norma IS011620, e como meta a eleição de um subconjunto dos mesmos para implementação na primeira fase do projeto. Os indicadores foram escolhidos em função do esforço necessário para coleta, consolidação e análise em face do tempo de resposta requerido pelos tomadores de decisão. A eleição dos indicadores levou também em conta a aderência com àqueles já em uso nas bibliotecas propiciando, assim, uma resposta mais rápida ao planejamento institucional.

Para a dinâmica da oficina foi adotada a formação de grupos heterogêneos de trabalho no que diz respeito ao desempenho das funções dos participantes, enriquecendo as discussões e compartilhando diferentes visões sobre um mesmo processo. A análise dos indicadores envolveu a conceituação e métricas disponíveis, aplicabilidade e eficácia teórica, oportunidade e propriedade de aplicação no ambiente Fiocruz e possíveis e prováveis adequações necessárias para implementação.

Como resultado foram escolhidos cinco indicadores para aplicação imediata: satisfação do usuário, visitas per capita à biblioteca, índice de uso de documentos, disponibilidade das instalações e índice de ocupação dos assentos. Essa escolha baseou-se no fato de que as metodologias indicadas não apresentariam dificuldades de entendimento e coleta de dados por parte dos profissionais. Evidenciou-se ainda no curso da oficina a importância de rever formas de atuação e de adotar mecanismos que viessem a possibilitar melhor desempenho e avaliação de alguns serviços. Ficou clara, também, a importância de uniformizar dados encaminhados para o planejamento central da Fiocruz, responsável pelo orçamento institucional.

O relatório gerado pela oficina foi chancelado pelas chefias das Bibliotecas e encaminhado à direção do Cict. As lacunas e inconsistências foram objeto de atenção na etapa seguinte do projeto. 
Dos indicadores selecionados, o primeiro a ser trabalhado foi 0 satisfação do usuário, que envolveu a delimitação do público-alvo de cada biblioteca e, em seguida, a identificação dos pontos a incluir no instrumento de coleta. Essa etapa envolveu esforço considerável por parte das bibliotecas, especialmente no que diz respeito às particularidades de cada usuário. Foi desenvolvido um questionário-padrão para coleta de dados cujo desenho foi baseado naquele descrito no Relatório do PAQ (SAMPAIO, 2004).

Numa escala de quatro níveis, o usuário deverá assinalar o grau de satisfação acerca dos seguintes aspectos das bibliotecas: condições ambientais, qualidade do acervo, serviços oferecidos, atitudes dos profissionais no atendimento ao usuário, qualidade (precisão, atualização e rapidez) da informação prestada, e critérios para utilização dos recursos informacionais. Parte do questionário é específica para tratar de aspectos particulares a alguma biblioteca, por exemplo, existência de salão de leitura e acervo de vídeos.

Uma vez elaborado o instrumento, foi realizado um teste piloto por cada uma das bibliotecas e procedidas às correções julgadas necessárias. A experiência possibilitou avaliar o impacto do instrumento junto aos usuários, no que tange à sua compreensão e à clareza dos pontos avaliados, bem como identificar lacunas. Na fase atual do projeto o instrumento está sendo implementado.

Em paralelo, estão em curso discussões iniciais sobre os outros indicadores selecionados, quais sejam, visitas per capita à biblioteca, índice de uso de documentos, disponibilidade das instalações e índice de ocupação dos assentos, especialmente no que toca a forma de coleta de informações para subsidiar a aplicação dos mesmos.

\section{0 futuro}

Confrontadas com os condicionantes técnico-científicos, socioeconômicos e culturais característicos do final do século XX, as bibliotecas não mais podem retardar a decisão de implantar programas de avaliação de desempenho. Especialmente para as instituições públicas, e em face de orçamentos financeiros cada vez mais modestos, conceitos como transparência, responsabilidade, accountability, efetividade e inclusão social/digital colocam aos gestores de bibliotecas desafios nunca antes considerados. O alinhamento com orientações estratégicas institucionais, a necessária priorização na alocação de recursos, o foco no cliente, a revolução das tecnologias de informação e comunicação e a conseqüente virtualização dos acervos e autonomia dos usuários são algumas das perspectivas que tornam imprescindíveis respostas sobre o impacto/desempenho/valor/qualidade dos serviços das bibliotecas.

Muito se progrediu nas últimas décadas no desenvolvimento de metodologias de avaliação de desempenho. Tal desenvolvimento, entretanto, não camufla a fluidez e subjetividade inerentes ao processo de avaliação e ao conceito de desempenho. De fato, especialistas na área são 
unânimes em enfatizar que dispor de metodologias adequadas é condição necessária, mas não suficiente para fazer da avaliação um instrumento efetivo e eficaz para a gestão das bibliotecas. Cullen (1997) lembra que décadas de estudos e aprendizado sobre avaliação, e dezenas de metodologias robustas disponíveis, não substituem o foco principal do sucesso de toda avaliação: liderança profissional e mudança de cultura organizacional. Outros autores vão mais longe (CRAMM, 1999) e alertam que só aquelas bibliotecas que já cultuem uma cultura de avaliação são capazes de levar adiante uma prática de avaliação de desempenho. Por cultura de avaliação entenda-se uma cultura de prática reflexiva cotidiana, que independe de metodologias formatadas, e está intrinsecamente ligada ao compromisso que liga o profissional à instituição, ao usuário, aos colegas de trabalho e às boas práticas profissionais.

As etapas subseqüentes do presente projeto, já planejadas e organizadas em um cronograma, apontam para a continuidade da pesquisa e um bom termo no desenho de uma metodologia para avaliação de desempenho das bibliotecas. O que o futuro não deixa claro, entretanto, é se o processo de aprendizagem vivenciado até aqui será suficiente para que as bibliotecas espelhem as mudanças esperadas (e defendidas) por muitos.

A clareza sobre essa realidade, e o envolvimento ativo dos profissionais bibliotecários no projeto são ingredientes essenciais para motivá-los a levar adiante a bandeira da importância decisiva que cabe aos serviços de informação em um contexto de pesquisa. A partir daí, o conceito de qualidade passará a ser (re)construído cotidianamente com o usuário e o tomador de decisão.

\section{Referências}

CAMILE. Concerted Action on Management Information for Libraries in Europe. Disponível em http://www.staff.dmu.ac.uk/ camile/. Acesso em 01 ago. 2005.

CARBONE, $P$. Évaluer la performance des bibliothèques : une nouvelle norme. $B B F$, Paris, n. 6, p. 40-45, 1998. Disponível em: <http://bbf.enssib.fr/sdx/BBF/frontoffice/1998/06/document.xsp?id=b bf-1998-06-0040-005/1998/06/fam-propos/apropos\&statutMaitre

$=$ non\&statutFils=non $>$. Acesso em 30 maio 2005.

CRAMM, J. Six impossible things before breakfast:a multidimensional approach to measuring the value of libraries. In: NORTHUMBRIA INTERNATIONAL CONFERENCE ON PERFORMANCE MEASUREMENT IN LIBRARIES AND INFORMATION SERVICES, 3., 1999, Newcastle. Proceedings... Newcastle, 1999.

CULLEN, R. Does performance measurement improve organisational effectiveness? A post-modern analysis' in Department of Information and Library Management, University of Northumbria at Newcastle. In: 
NORTHUMBRIA INTERNATIONAL CONFERENCE ON PERFORMANCE MEASUREMENT IN LIBRARIES AND INFORMATION SERVICES, 2., 1997, Newcastle. Proceedings... Newcastle, 1998.

INTERNATIONAL FEDERATION OF LIBRARY ASSOCIATIONS AND INSTITUTIONS. International Guidelines for Performance Measurement in Academic Libraries. 1996. Disponível em http://www.ifla.org/V/saur.htm. Acesso em 01 ago. 2005.

INTERNATIONAL ORGANIZATION FOR STANDARDIZATION: ISO 9004-2 Guidelines for managing the quality of service activities. Genève: ISO, 1991.

: ISO 11620/98 Information and documentation library performance indicators. Genève: ISO; $2003 a$.

: ISO 2789 Information anddocumentation - library performance. Genève: ISO, 2003b.

LANCASTER, F. W. Avaliação de serviços de Biblioteca. Brasília: Briquet de Lemos, 1996.

LIBQUAL+ ${ }^{\mathrm{TM}}$. Defining and promoting library service quality. Disponível em http://www.libqual.org/About/Information/index.cfm. Acesso em 29 jul. 2005.

MANDANI, Y. The Linnean Society of London and its library. London: Uppsala University, 2004.

MELO, L. B. Estatísticas e avaliação da qualidade e do desempenho em bibliotecas e serviços de informação: investigações recentes e novos projectos. In: CONGRESSO NACIONAL DE BIBLIOTECÁRIOS, ARQUIVISTAS E DOCUMENTALISTAS, 8., 2004, Lisboa. Anais... Lisboa: APBAD, 2004. Disponível em http://eprints.rclis.org/archive/ 00004156/01/EstAvalBSI_LM_2004.pdf Acesso em 17 jul. 2005.

PARASURAMAN, A.; ZEITHAML, V. A.; BERRY, L. L. A conceptual model of service quality and its implications for future research. Journal of Marketing, v. 49, p. 41-50, 1985.

ROZADOS, H. B. F. Uso de indicadores na gestão de recursos de informação. Revista digital de biblioteconomia e ciência da informação, v. 3, n. 1, p. 60-76, 2005. Disponível em: http://server01.bc.unicamp.br/seer/ojs/viewarticle. php?id=41\&layout= abstract. Acesso em 10 jul. 2005.

SAMPAIO, M. I. C. et al. PAQ : Programa de avaliação da qualidade de produtos e serviços de informação:uma experiência no SIBI/USP. Ciência da Informação, v. 33, n. 1. p. 142-148, 2004. 
Indicadores de desempenho de bibliotecas no campo da saúde: relato de estudo na Fiocruz
Maria Cristina S. Guimarães; Etiene Gonzalez Lins; Jeorgina Gentil Rodrigues; Camila Clementino Lamarão; Márcia Jabor Santos; Vânia Guerra da Silva; Diones Ramos da Silva

SERVQUAL: measuring service quality in academic libraries. 1997. Disponível em http://www.arl.org/newsltr/191/servqual.html. Acesso em 29 jul. 2005. 